\title{
EFFECT OF DESKTOP SCANNING PROTOCOL ON ACCURACY OF GUIDED IMPLANT PLACEMENT IN COMPLETELY EDENTULOUS PATIENTS
}

\author{
Ahmed Mostafa Abdelfattah*
}

\begin{abstract}
Purpose: The purpose of this study was to evaluate the effect of Desktop scanning protocol on accuracy of guided implant placement in completely edentulous patients.

Methodology: Twenty four completely edentulous patients were selected for implant placement. Complete dentures were made for all the patients. Radioopaque markers were added to the dentures to be used as a radiographic stent. A Cone beam CT scan was then made for each patient while wearing the radiographic stent. The patients were then randomly divided into 2 equal groups. In the first group, a CBCT scan was made for the radiographic stent. In the second group, desktop scanning was made for the radiographic stent while being seated on the patient's cast and another scan for the patient's cast. After implant insertion, CBCT scan was made for each patient while wearing the radiographic stent. Superimposition of CBCT scans pre and post implant insertion was made. Deviations between planned and placed implant were measured in the lateral apical, lateral coronal and angular aspects.
\end{abstract}

Results: Non statistically significant difference between radiographic and desktop scanning on implant deviation was observed.

Conclusion: within limitation of this study, effect of both scanning protocols on the lateral apical, lateral coronal and angular deviations of placed implants is comparable.

KEYWORDS: Desktop scanning, surgical guides, Completely edentulous patients, Deviation, Implants

\section{INTRODUCTION}

Digital planning of implant placement improves functional outcome. Thanks to recent advances in cone-beam computed tomography (CBCT), different scanning protocols, CAD softwares, be guaranteed by the help of guided implant surgery. milling machines and $3 \mathrm{D}$ printers that have facilitated the transition from traditional treatment planning to a complete digital implant workflow. ${ }^{(1)}$

Accurate and precise implant placement can now

\footnotetext{
* Lecturer, Oral and Maxilofacial Prosthodontics Department, Faculty of Dentistry, AinShams university, Cairo, Egypt
} 
Moreover, surgical guides allow conservative flapless surgery. Also, surgical complications are limited and the operating time is shortened. Patient satisfaction is furtherly improved too. ${ }^{(2)}$

Accuracy in guided implant surgery can be evaluated by measuring the difference between the implant's planned position with its final position. The accuracy of implant position is dependent on the errors that take place during data acquisition with cone-beam computed tomography (CBCT), data processing, accuracy of the $3 \mathrm{D}$ printer, placement of the guide during surgery and its movement during drilling. ${ }^{(3)}$ Moreover, accuracy of the guide is also dependent on implant site location, whether the patient being completely or partially edentulous, the type of tissue support for the guide and the amount of surgical restriction offered by it. . $^{(4-6)}$

Surgical guides for partially edentulous patients were found to be more accurate than completely edentulous patients. Furthermore, surgical guides supported by posterior teeth compared to anterior teeth, showed a higher level of accuracy. ${ }^{(7)}$ Surgical guides can be classified according to type of tissue support into bone supported, mucosa supported and teeth supported surgical guides. Bone- supported guides were found to have greater deviation compared with teeth or mucosa- supported guides. However, no differences in implant position accuracy were recorded between mucosa- and teeth-supported guides. ${ }^{(8)}$

Surgical guides can be also classified according to the amount of surgical restriction offered by the surgical guide design into complete limiting and partial limiting guides. In partial limiting surgical guides part of the drilling sequence is made through the guide. However, in completely limiting surgical guides the whole drilling sequence is made through the guide. ${ }^{(8)}$.Although completely limiting surgical guides showed higher accuracy than partially limiting surgical guides yet, there was no statistically significant difference between them as concluded from further studies. ${ }^{(3,8)}$

Two CBCT scan protocols are usually used for manufacturing of surgical guides in completely edentulous patients. The first CBCT scan is made for the patient while wearing the radiographic stent and a second scan is made for the radiographic stent itself. With recent advances in digital optical scanning, one CBCT scan can be made for the patient while wearing the radiographic stent. Two digital scans can then be made by desktop scanner; one for the dental model alone and the second one for the dental model with the radiographic stent seated on it. .9-11) $^{-11}$

The aim of this study was to evaluate the effect of desktop scanning protocol on accuracy of guided implant placement in completely edentulous patients compared to the conventional radiographic scanning protocol. The null hypothesis that there was no difference between both scan protocols was accepted in this study.

\section{MATERIALS AND METHODS}

\section{Sample size selection and grouping:}

Twenty four patients were selected to share in this study. G power program (3. 1) was used for calculations of sample size. Statistical calculator was based on $95 \%$ confidence interval and power of the study $80 \%$ with $\alpha$ error $5 \%$. According to a previous study (Fully Digital Workflow for Planning Static Guided Implant Surgery, Lin CC et al.), the mean of angular deviation was $4.96^{\circ} \pm 3.32$ in one group compared to the second group that was $3.38^{\circ} \pm 1.81$. For lateral apical deviation, it was $1.34 \mathrm{~mm} \pm 0.86$, $0.87 \mathrm{~mm} \pm 0.3$ in both groups, and $0.64 \mathrm{~mm} \pm 0.36$, $0.47 \mathrm{~mm} \pm 0.26$ for lateral coronal deviation. Using un paired t test the results were found to be insignificant. Based on this study, sample size was calculated according to these values producing a minimal sample size of twenty four patients being enough to find such a difference. 
The patients were randomly divided into two equal groups; group (I) and group (II). Randomization was guided by a computer generated list. Blinding was done by one of the postgraduate students who was the only one to know the key so that the authors and the patients did not know in which group the patient was. Afterwards, the authors were informed by the randomization to be tabulated.

Ethical approval was granted by the research ethics committee in the Faculty of Dentistry Ain Shams university (FDASUREC). All patients were informed in details about the nature of investigation and the aim of the study. Patients' data were kept safe and confidential to protect the security and privacy of the patient information. All participants were given notice about their privacy and their rights.

\section{Patient selection}

The patients were selected according to the following criteria: completely edentulous patients, non-smokers, 50-70 years in age and with no previous history of radiotherapy. Patients were also free from systemic diseases that may affect osseointegration as uncontrolled diabetes mellitus. Moreover, patients were free from systemic diseases that might affect bone quality, increase surgical risk, delay or complicate post-operative healing. Good oral hygiene and motivation were mandatory in all selected patients. Intra oral examination was performed to ensure firm healthy mucosa covering the residual ridge, free from any signs of flabbiness, ulceration or inflammation. Presence of adequate keratinized mucosa was essential in all cases. Absence of any pathologic lesion was ensured and salivary secretion was examined for rate and consistency.

\section{Surgical guide designing and construction:}

All patients received maxillary and mandibular complete dentures that were made in a conventional way. Radioopaque markers were added to each patient's mandibular denture to be used as a radiographic stent. A Cone Beam CT scan(Icat Gendex kavo,CA,USA) was made for each patient while wearing the radiographic stent.

For patients in group I, another CBCT scan was made for the radiographic stent. For each patient, the DICOM files were exported to Blue Sky implant designing software (Langenhagener, Mdi Europa $\mathrm{GmbH}$ ) for superimposition of landmarks in both scans. However, for patients in group II, the radiographic stent was digitally scanned using a desktop scanner (Medit digital scanner, Seoul, South Korea) while being seated on the cast in addition to another digital desktop scan for the cast alone. $\mathbf{F i g}(\mathbf{1})$ The STL files of both scans were exported to Blue sky software and superimposed. The DICOM files of the CBCT scan for the patient while wearing the radiographic stent were exported also to the Blue sky software. Superimposition of the digital scans and the CBCT scan was then done. The dimensions of each implant were determined at the proposed implant sites; mandibular canine position. The implant position and angulation were virtually designed. Two virtual implants of appropriate length and diameter were placed into their proposed sites and parallelism between them was considered. Fig(2) Channels that should receive the metallic sleeves later were designed on the Blue Sky Plan software. All the surgical guides were planned to be partially limiting in this study. The position of the anchor pins was determined (three pins, one in the midline and two posterior pins one on each side). Three channels corresponding to the desired position of the anchor pins were added during the virtual planning. Once the design was accepted, the STL file format was exported to the 3D printer for fabrication of the surgical guide. Fig(3) The 3D printed guide was autoclaved at a pressure of $+1 \mathrm{bar}$ and a temperature of $121{ }^{\circ} \mathrm{C}$ for 20 minutes. It was then checked in the patient's mouth. 


\section{Surgical procedures:}

The maxillary complete denture was inserted and seated in place. After checking the local anesthesia (Mepiccaine local anathesia, Alexandria company for pharmaceuticals and chemical industry); the surgical guide was inserted into the patient's mouth. During surgery, the surgical guide was placed in position and stabilized against the upper denture. Three osteotomies corresponding to the position of the anchor pins were made, pins were then secured in place and the guide was then checked for stability in the patient's mouth.

Each patient in each group received 2 endosteal interforaminal implant(NeoBiotech dental implants) to retain the mandibular denture. Intermittent drilling under copious irrigation using sharp drills was made to reduce heat generation. External refrigerated saline was also used during irrigation to control further heat generation. As the surgical guide was designed to be partially limiting in this study, the final drill of the implant manufacturer was the only drill to be used free hand .The implant was then inserted in the osteotomy site and the cover screws were then secured in place.

\section{Methods of evaluation}

Patients were recalled after implant insertion for another CBCT scan. The same pre-operative CBCT machine and parameters were used while the patient was wearing the radiographic stent
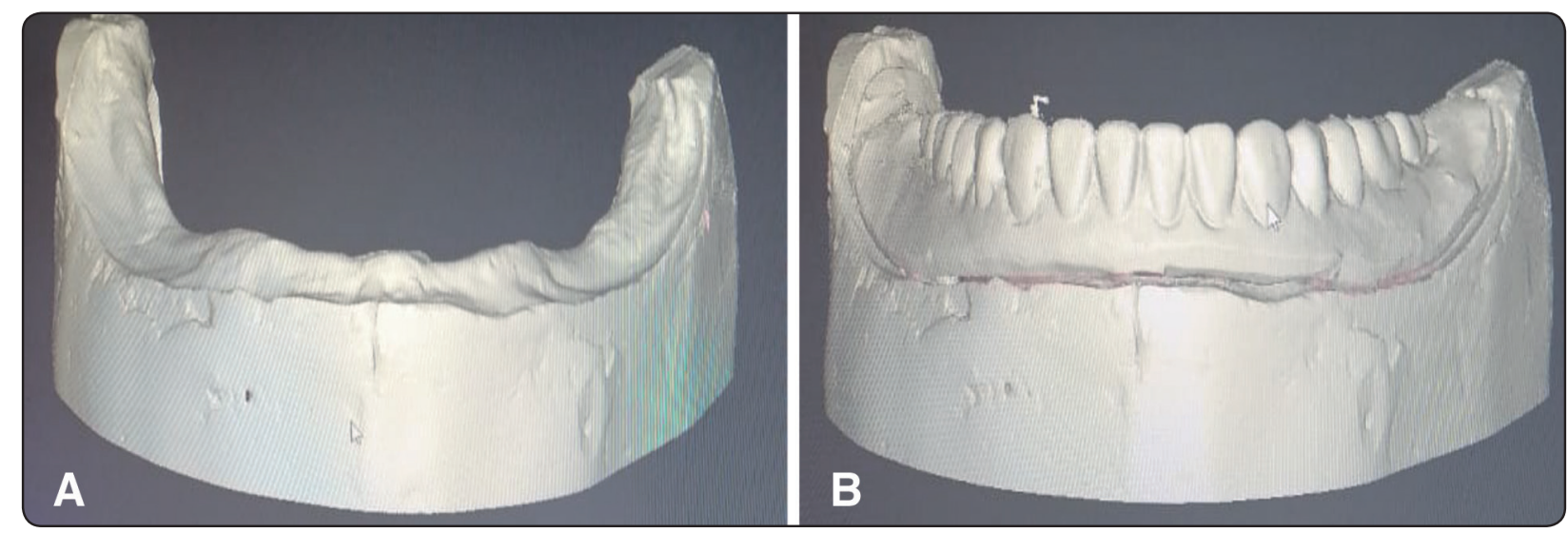

Fig (1-A): Digital desktop scan of the patient's cast. Fig (1-B): Digital desktop scan of the radiographic stent placed on the patient's cast.
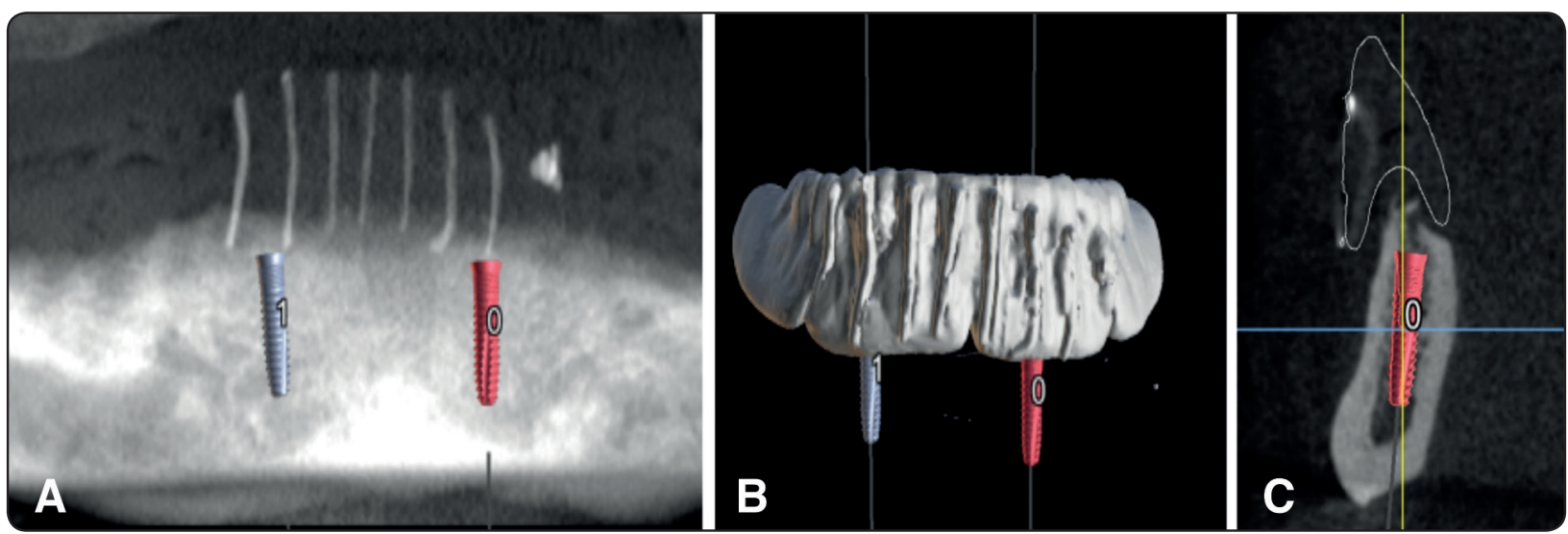

Fig (2-A): Implant sites planning in the bone. Fig (2-B): parallelism between proposed implant sites. Fig (2-c): Angulation of implant position 
and biting against the upper denture. The DICOM data were exported to the Blue Sky Plan software for segmentation; removal of soft tissue. Super imposition of pre and post operative scans was then made. The virtually planned and actual implant positions were compared. Three deviation parameters were used to evaluate the amount of deviation between the pre-operative planned and the actual implant positions; lateral coronal, lateral apical, and angular deviations. The lateral coronal and lateral apical deviations were measured in $\mathrm{mm}$ and the angular deviation in degrees. $\operatorname{Fig}(\mathbf{4})$

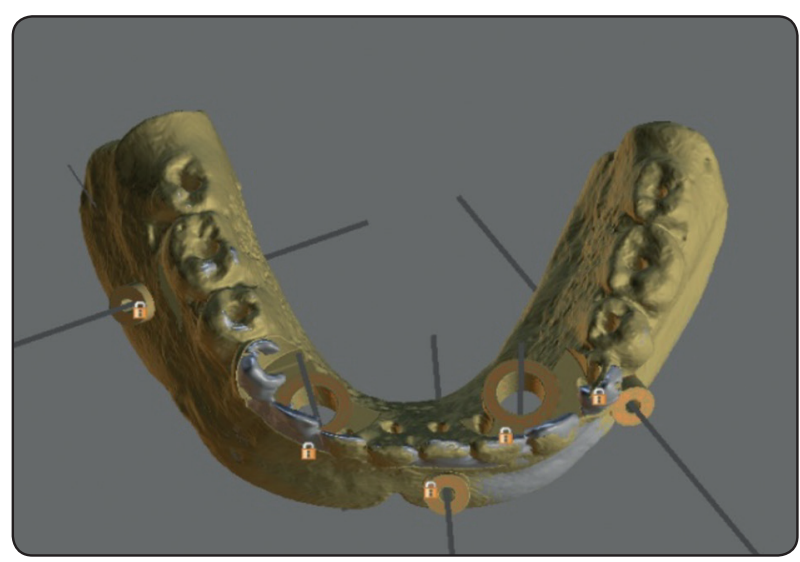

Fig (3): Final virtual design of the surgical guide showing position of anchor pins and implants ready for $3 \mathrm{D}$ printing
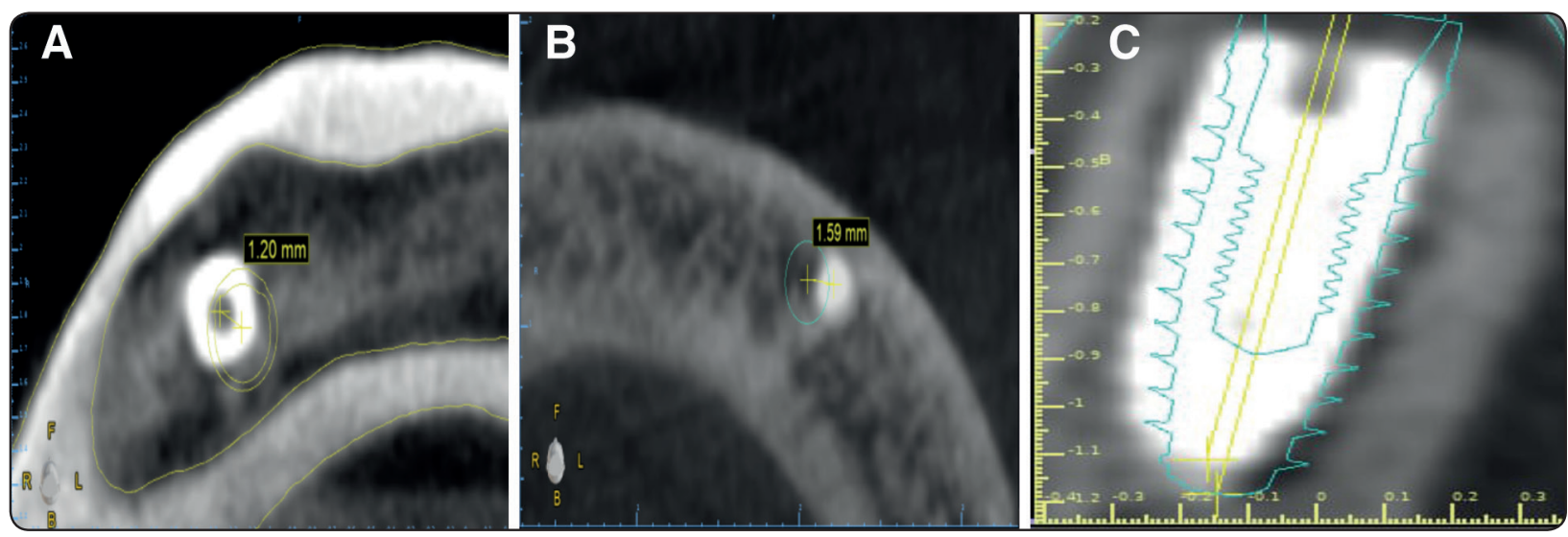

Fig (4-A): Measuring lateral coronal deviation. Fig (4-B): Measuring lateral apical deviation. Fig (4-c): Measuring angular deviation

\section{RESULTS}

The data for lateral coronal, lateral apical and angular deviations were recorded and statistically analysed. The data were tested for normality and uniform distribution using Shapiro-Wilk test. The data were found to be normally distributed. Independent- $\mathrm{t}$ - test was used to find out any statistically significant difference between both groups in any of the three deviation parameters. (table1, figure 5) The mean of group (I) regarding the lateral apical deviation was $1.05 \mathrm{~mm} \pm 0.54$ however for group (II) was $1.07 \mathrm{~mm} \pm 0.32$. The Pvalue was less than 0.05 indicating non statistically significant difference. However, the mean of group (I) regarding the lateral coronal deviation was $0.89 \mathrm{~mm} \pm 0.32$, while for group (II) was $0.87 \mathrm{~mm}$ \pm 0.48 . The $\mathrm{P}$-value was less than 0.05 indicating non statistically significant difference. Furthermore, the mean of group (I) regarding the angular deviation was $2.7^{\circ} \pm 0.73$ however for group (II) was $2.4^{\circ}$ \pm 0.77 . The P- value was less than 0.05 indicating non statistically significant difference. 
TABLE (1): Mean and standard deviation for implant parameters in both groups

\begin{tabular}{|l|c|c|c|}
\hline & Group (I) & Group (II) & p-value \\
\hline $\begin{array}{l}\text { Lateral apical deviation } \\
\text { Mean } \pm S D\end{array}$ & $1.05 \mathrm{~mm} \pm 0.54$ & $1.07 \mathrm{~mm} \pm 0.32$ & 0.93 \\
\hline $\begin{array}{l}\text { Lateral coronal deviation } \\
\text { Mean } \pm \text { SD }\end{array}$ & $0.89 \mathrm{~mm} \pm 0.32$ & $0.87 \mathrm{~mm} \pm 0.48$ & 0.94 \\
\hline $\begin{array}{l}\text { Angular deviation } \\
\text { Mean } \pm \text { SD }\end{array}$ & $2.7^{\circ} \pm 0.73$ & $2.4^{\circ} \pm 0.77$ & 0.41 \\
\hline
\end{tabular}

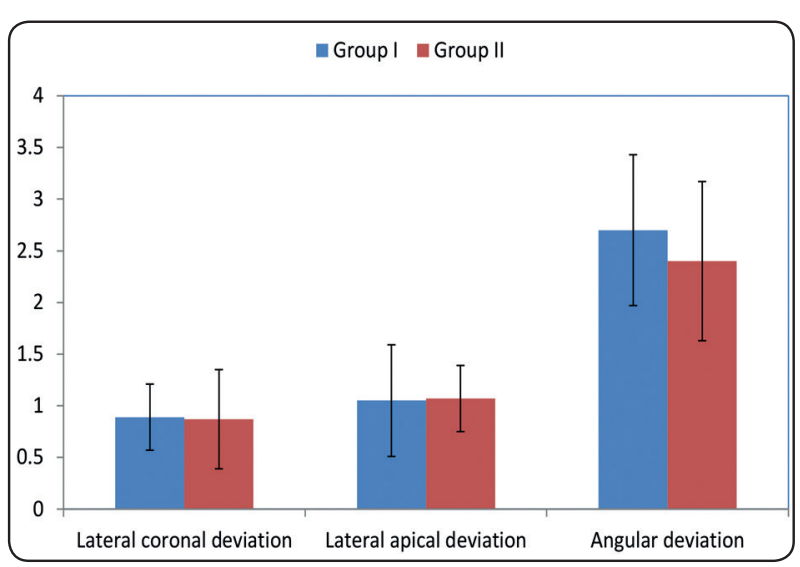

Fig (5): Bar chart showing mean and standard deviation values for lateral coronal, lateral apical and angular deviation in both groups

\section{DISCUSSION}

This study was a randomized clinical trial that evaluated the effect of desktop scanning on the accuracy of guided implant placement in completely edentulous patients compared to the conventional radiographic scanning protocol. The null hypothesis that there was no difference between both scanning protocols was accepted.

Mandibular implant assisted overdenture was the prosthetic superstructure used in this study. It was reported to offer lots of advantages as improved quality of life and patient satisfaction. Furthermore, implant overdenture is simpler and less expensive compared to implant supported fixed prosthesis. Easier hygiene access was also reported with implant assisted overdentures compared to implant supported fixed restorations. Also, the rate of bone height changes was reported to be less over ten-year follow up after implant placement compared with the rates seen with conventional dentures. ${ }^{(12)}$

Surgical guides help the implants to be placed in optimum position in the alveolar bone to enhance function and aesthetics. ${ }^{(13)}$ Moreover, guides help to place the implants with the desired angulation and parallelism. ${ }^{(14)}$ Furthermore, malaligned implants are coupled with improper load distribution, stress concentration, high rates of crestal bone loss, screw loosening and prosthetic components fracture. ${ }^{(15)}$

Several studies reported high survival rates of implants placed by surgical guides. ${ }^{(16)}$ Implants placed with a guide appear to have a survival rate comparable with those placed without a guide in further studies.(17-20) Developments in conebeam computed tomography (CBCT), scanning protocols, CAD softwares, milling machines and 3D printers have facilitated the transition from traditional treatment planning to a complete digital implant workflow. ${ }^{(1)}$

Surgical guides used in this study were designed to be mucosa supported as bone supported surgical guides were found to be less accurate compared to mucosa supported ones. ${ }^{(3,7)}$ In the present study, the surgical guides were partially limiting rather than completely limiting ones. No statistically significant differences were reported in the literature between 
both types of surgical guides. ${ }^{(3,8)}$ Partially limiting ones were used for better vision during final drilling and implant insertion.

Different scanning protocols can be used for the manufacturing of surgical guides; single, double and triple scan protocols. Multiple scanning protocols rather than single scanning protocols were used in this study. The single scan protocol was not used in this study as mucosa supported guides can not be manufactured using this protocol. Moreover, multiple scan protocols help to overcome drawbacks of single scan protocol as the loss of data related to the segmented bone during volume rendering. (21,22) In group I, a CBCT scans was made for the radiographic stent. The DICOM files of the CBCT scans of the patient and the stent were imported into Blue Sky Software for superimposition of both scans and designing the guide. Superimposition was done by registering landmarks in both scans. ${ }^{(22)}$

In group II two desktop scans were made; one for the patient's cast alone and the other one for the patient's cast with the radiographic stent over it. Superimposition of these scans was done and then registering landmarks between the CBCT scan and the superimposed desktop scans. ${ }^{(23)}$ Desktop scanning for the cast was preferred to intra oral scanning as desktop scanning was reported to be more accurate in terms of trueness and precision than intraoral scanning in completely edentulous patients. ${ }^{(24)}$

After implant designing and planning, the STL file was exported to a $3 \mathrm{D}$ printer for production of the surgical guide. 3D printed surgical guides were used in this study as degrees of deviation obtained by $3 \mathrm{D}$ printed surgical guides were found to be comparable to milled surgical guides as reported in the literature. ${ }^{(25)}$ Furthermore, milling manufacturing technique is very wasteful as a lot of material is removed compared to the $3 \mathrm{D}$ printing technology. ${ }^{(26)}$ The surgical guide was then autoclaved at +1 bar pressure and $121^{\circ} \mathrm{C}$ for
20 minutes as accuracy of surgical guides is not affected when sterilized under such conditions. ${ }^{(27)}$ It was then seated in place and stabilized against the upper denture. ${ }^{(28)}$ Fixation of the guide then took place by the help of the anchor pins for guide stabilization during surgical procedures to minimize implant deviation. ${ }^{(29)}$ Drilling was intermittent and under copious irrigation to reduce heat generation; a problem encountered while using surgical guides. Furthermore, external irrigation was also used and the irrigating saline was refrigerated. Heat generation during osteotomy preparation may result in a zone of devitalized bone around the osteotomy; a condition that may affect implant osseointegration. (30,31) This can be explained due to the fact that survival of the bone cells is highly influenced by the amount of heat generated during drilling. ${ }^{(32)}$ Drilling was also carried out in an up and down motion to allow irrigation to pass through the metallic sleeves and soft tissue to reach the osteotomy site and provide sufficient cooling. Further precautions to reduce heat generation were taken in both groups as intermittent drilling, decreased drill speed, sharp drills and regular withdrawal of the drill. ${ }^{(33)}$

Postoperative CBCT scan was made for each patient while wearing the radiographic stent. The patients were instructed to bite against the upper denture .This helped to seat the radiographic stent in a position similar to that of the preoperative position allowing for accurate superimposition of preoperative and postoperative DICOM files. ${ }^{(34)}$ Pre and post operative DICOM files were exported to Blue Sky software and superimposed using landmarks in the radiographic stent. Deviations between the planned and placed implants were measured in the lateral apical, lateral coronal and angular aspects similar to previous studies. ${ }^{(35,36)}$

Results of this study show statistically insignificant difference between both groups in the three aspects of deviation. The mean values of lateral coronal, lateral apical and angular 
deviations for both groups are within the safety margin mentioned in the literature; 1-1.5 mm. . $^{(37)}$ Similarly, the average mean values in this study come in line with the mean values of another studies that compared different optical scanning protocols to the conventional radiographic one. $(11,22,38,39)$ Comparable results between both groups in this study could be related to the findings of several studies which showed that superimposition of optical and radiographic scans have the same accuracy. ${ }^{(40-43)}$ Moreover, high accuracy up to $10 \mathrm{um}$ can be attained by desktop scanners. ${ }^{(44)}$ In addition, the factors that affect accuracy of guided implant placement were controlled between both groups. ${ }^{(3)}$ The CBCT machine and parameters, positions of radiopaque markers, planning software and same printing machine were unified for patients in both groups.

\section{CONCLUSION}

Within the limitation of this study, effect of both scanning protocols on the lateral apical, lateral coronal and angular deviations of placed implants is comparable.

\section{Study limitations}

Limitations of this study involved the inherent errors that might have occurred during the study resulting from data acquisition with cone-beam computed tomography (CBCT), data processing, accuracy of the $3 \mathrm{D}$ printer, placement of the guide during surgery, its movement during drilling in addition to superimposition of pre and post operative CBCT scans.

\section{Research recommendations}

With recent advances in intraoral scanning devices and techniques, it can be recommended to use intraoral scanning technique instead of desktop scanning technique to assess its efficacy as a scanning protocol. Moreover, further methods to determine implant position and angulation postoperatively can be used rather than $\mathrm{CBCT}$ as intraoral scanning; a condition that helps to prevent patients from further exposure to radiation.

\section{Conflict of interest}

No conflicts of interest to be declared in this study.

\section{REFERENCES}

1. Walker-Finch K. and Ucer C. Five-year survival rates for implants placed usingdigitally-designed static surgical guides: a systematic review. Brit $\mathbf{J}$ of Oral and Maxillofac Surg 2020;58: 268-76.

2. Yeung M, Abdulmajeed A, Carrico C K, Deeb G, and Bencharit S. Accuracy and precision of 3D-printed implant surgical guides with different implant systems: An in vitro study. J Proth Dent 2020;123:821-8.

3. Monaco C., Arena A., Corsaletti L., Santomauro V., Venezia P., Cavalcanti R et al. 2D/3D accuracies of implant position after guided surgery using different surgical protocols: A retrospective study. J Prosth Res 2020; 64: 424-30.

4. Naeini E N, Atashkadeh M, De Bruyn H and D'Haese J. Narrative review regarding the applicability, accuracy and clinical outcome of flapless implant surgery with or without computer guidance. J Clin Implant Dent Relat Res 2020;22:454-67.

5. Jia P, Yang G, Hu W, Chung KH, Zhao Y, Liu M et al. Comparison of in Situ Cone Beam Computed Tomography Scan Data with Ex Vivo Optical Scan Data in the Measurement of Root Surface Area. Oral Surg, Oral Med, Oral Pathol, and Oral Radiol 2019; 128: 552-7.

6. Liu Y, Wu J, Zhang J, Peng W and Liao W. Numerical and Experimental Analyses on the Temperature Distribution in the Dental Implant Preparation Area when Using a Surgical Guide. J of Prosthodontics 2018; 27: 42-51.

7. El Kholy K, Lazarin R, F. M. Janner S, Faerber K, Buser $\mathrm{R}$ and Buser D. Influence of surgical guide support and implant site location on accuracy of static ComputerAssisted Implant Surgery. J Clin Oral Impl Res 2019; 30: $1067-75$.

8. Tattan M, Chambrone L, MartínO G and Ortiz GA. Static computer-aided, partially guided, and free-handed implant placement: A systematic review and meta-analysis 
of randomized controlled trials. Clin Oral Impl Res. 2020;31:889-916.

9. Kernen F, Kramer J, Wanner L, Wismeijer D, Nelson K and Flügge T. A review of virtual planning software for guided implant surgery - data import and visualization, drill guide design and manufacturing. J BMC Oral Health 2020;20:251-60.

10. Lal K, White GS, Morea DN and Wright RF. Use of stereolithographic templates for surgical and prosthodontic implant planning and placement. Part I. The concept. J Prosthodont. 2011;15(1):51-8.

11. Pettersson A, Komiyama A, Hultin M, Näsström $K$ and Klinge B. Accuracy of virtually planned and template guided implant surgery on edentate patients. Clin Implant Dent Relat Res. 2012;14(4):527-37.

12. Passia N, Wolfart S and Kern M. Ten-year clinical outcome of implant-retained mandibular overdentures-A prospective pilot study. J Dent. 2019;82:63-5.

13. Kattadiyil MT, AlHelal A and Goodacre BJ. Clinical complications and quality assessments with computerengineered complete dentures: A systematic review. J Prosthet Dent. 2017;117(6):721-8.

14. Hinckfuss S, Conrad HJ, Lin L, Lunos S and Seong WJ. Effect of surgical guide design and surgeon's experience on the accuracy of implant placement. J Oral Implantol. 2012;38(4):311-23.

15. Chackartchi T, Romanos GE and Sculean A. Soft tissuerelated complications and management around dental implants. J Periodontol 2000. 2019; 81: 124- 38.

16. Laleman I, Bernard L, Vercruyssen M, Jacobs R, Bornstein MM and Quirynen M. Guided implant surgery in the edentulous maxilla: a systematic review. Int J Oral Maxillofac Implants 2016;31:103-17.

17. KimWH., Lee J C., Lim D., Heo Y. K., Song E S., Lim YJ et al. Optimized Dental Implant Fixture Design for the Desirable Stress Distribution in the Surrounding Bone Region: A Biomechanical Analysis. J. Materials 2019; 12:2749.

18. TangT, Liao L., Huang Z., Gu X., and Zhang, X. Accuracy of the evaluation of implant position using a completely digital registration method compared with a radiographic method. J Prosth Dent 2019;122: 537-42.

19. Vercruyssen M, Cox C, Naert I, Jacobs R, Teughels W, and Quirynen M. Accuracy and Patient-centered Outcome
Variables in Guided Implant Surgery: A RCT Comparing Immediate with Delayed Loading. J Clini Oral Impl Res 2016;27: 427-32.

20. Moraschini V, Vellosa G, Luz D and Barboza EP. Implant survival rates, marginal bone level changes and complications in full mouth rehabilitation with flapless computer guided surgery: a systematic review and meta analysis. Int J Oral Maxillofac Surg2015;44:892-901.

21. Kiatkroekkrai P, Takolpuckdee C, Subbalekha K, Mattheos $\mathrm{N}$ and Pimkhaokham A. Accuracy of implant position when placed using static computer-assisted implant surgical guides manufactured with two different optical scanning techniques: a randomized clinical trial. Int J Oral Maxillofac Surg. 2020;49:377-83.

22. Tallarico M, Xhanari E, Kim YJ, Cocchi F, Martinolli M, Alushi A et al. Accuracy of computer-assisted templatebased implant placement using conventional impression and scan model or intraoral digital impression: A randomised controlled trial with 1 year of follow-up. Int J Oral Implantol. 2019;12:197-206.

23. Pettersson A, Komiyama A, Hultin M, Näsström K and Klinge B. Accuracy of virtually planned and template guided implant surgery on edentate patients. Clin Implant Dent Relat Res. 2012;14:527-37.

24. Greenberg AM. Digital technologies for dental implant treatment planning and guided surgery. Oral Maxillofac Surg Clin North Am. 2015;27:319-40..

25. Henprasert P, Dawson DV, El-Kerdani T, Song X, Queiruga EC and Holloway J. Comparison of the Accuracy of Implant Position Using Surgical Guides Fabricated by Additive and Subtractive Techniques. Journal of Prosthodontics 2020;29: 534-41.

26. D'haese J, Van De Velde T, Komiyama A, Hultin M and De Bruyn H. Accuracy and complications using computerdesigned stereolithographic surgical guides for oral rehabilitation by means of dental implants: a review of the literature. J Clin Implant Dent Relat Res. 2012;14:321-35.

27. Török G, Gombocz P, Bognár E, Nagy P, Dinya E, Kispélyi B et al. Effects of disinfection and sterilization on the dimensional changes and mechanical properties of 3D printed surgical guides for implant therapy - pilot study . J BMC Oral Health 2020; 20:19-31.

28. Vinci R, Manacorda YM, Abundo YR, Lucchina A G, Scarano A, Crocetta C et al. Accuracy of Edentulous Computer-Aided Implant Surgery as Compared to Virtual 
Planning:A Retrospective Multicenter Study. J. Clin. Med. 2020; 9:774-84.

29. Apostolakis D and Kourakis G. CAD/CAM implant surgical guides: maximum errors in implant positioning attributable to the properties of the metal sleeve/osteotomy drill combination. Int J Implant Dent 2018;4:34.

30. Nilay E, Alkan A, Ilday S and Bengu E. Improved Dental Implant Drill Durability and Performance Using Heat and Wear Resistant Protective Coatings. J Oral Implantology 2018;44:168-75.

31. Montero-Aguilar A, Sibaja-Ruiz L, Avendaño E, PereiraReyes R, Pozos-Guillén A and Chavarria-Bolaños D. Evaluation of Surgical Bur Deformation and Bone Surface Roughness after Multiple Uses. J Oral and Maxillofac Surg2019; 77:1894-903.

32. Oh HJ, Kim BI, Kim HY, Yeo IS, Wikesjö UM and Koo KT. Implant Drill Characteristics: Thermal and Mechanical Effects of Two-, Three-, and Four-Fluted Drills. Int J Oral Maxillofac Implants. 2017;32:483-8.

33. Barrak I, Boa K , Joób-Fancsaly A, Varga E, Sculean A and Piffkó J. Heat Generation During Guided and Freehand Implant Site Preparation at Drilling Speeds of 1500 and 2000 RPM at Different Irrigation Temperatures. Oral Health Prev Dent 2019;17:309-16.

34. Marliere, D.A.A, Demetrio M.S, Picinini, L.S, De Oliveira R.G and Chaves Netto, Accuracy of computer-guided surgery for dental implant placement in fully edentulous patients: A systematic review. Eur. J. Dent. 2018; 12,: 153-60.

35. Van Assche $\mathbf{N}$, van Steenberghe D, Quirynen $\mathrm{M}$ and Jacobs R. Accuracy assessment of computer-assisted flapless implant placement in partial edentulism. J Clin Periodontol. 2010;37:398-403.

36. Sun TM, Lee HE and Lan TH. Comparing Accuracy of Implant Installation with a Navigation System (NS), a Laboratory Guide (LG), NS with LG, and Freehand Drilling. Int. J. Environ. Res. Public Health 2020; 17: 2107-21.
37. Raico Gallardo YN, da Silva-Olivio IRT and Mukai E. Accuracy comparison of guided surgery for dental implantsaccording to the tissue of support: a systematic review andmeta-analysis. Clin Oral Implants Res 2017;28:602-12.

38. Ben Yehuda D, Weber HP, Finkelman M, Sicilia E, Muftu A, Chochlidakis K et al. Accuracy of Guided Implant Surgery in 25 Edentulous Arches: A Laboratory Observational Study. J Prosth 2020;29:718-24.

39. Lin CC, Wu CZ, Huang MS, Huang CF, Cheng HC and Wang DP. Fully Digital Workflow for Planning Static Guided Implant Surgery: A Prospective Accuracy Study. J. Clin. Med. 2020; 9: 980-95.

40. Widmann, G., Berggren, J.Pand Fischer, B. PichlerDennhardt, A.R.; Schullian, P.; Bale, R.; Puelacher, W. Accuracy of Image-Fusion Stereolithographic Guides: Mapping CT Data with Three-Dimensional Optical Surface Scanning. Clin. Implant Dent Relat. Res. 2015; 17: 736-44.

41. Schnutenhaus, S.; Groller, S.; Luthardt, R.G and Rudolph, H. Accuracy of the match between cone beamcomputed tomography and model scan data in template-guided implant planning: A prospective controlled clinical study. Clin. Implant Dent. Relat. Res. 2018;20:541-9.

42. Suksod N, Kunavisarut C and Kitisubkanchana J. Accuracy of computer-guided implantation in the placement of onepiece ceramic dental implants in the anterior region: A prospective clinical study. J Plos One2020;15:1-17.

43. Schneider D, Sancho-Puchades M, Mir-Marí J, Mühlemann S, Jung R and Hämmerle C. A Randomized Controlled Clinical Trial Comparing Conventional and Computer-Assisted Implant Planning and Placement in Partially Edentulous Patients. Part 4: Accuracy of Implant Placement. Int J Periodontics Restorative Dent. 2019;39:111-22.

44. Siqueira R, Chen Z, Galli M, Saleh I, Wang H-L, Chan H-L. Does a fully digital workflow improve the accuracy of computer-assisted implant surgery in partially edentulous patients? A systematic review of clinical trials. ClinImplant Dent Relat Res. 2020;22:1-12. 